\title{
ON RADIO EMISSION AND RELATED X-RAYS IN \\ SOLAR-LIKE STELLAR CORONAE
}

\author{
M. GÜDEL \\ Paul Scherrer Institute \\ CH-5232 Villigen PSI, Switzerland
}

\begin{abstract}
Selected results relevant to coronal structuring in solar-like single stars based on combined radio and $\mathrm{X}$-ray information are presented. Nonthermal radio and thermal soft $\mathrm{X}$-ray emissions from coronal solarlike stars provide direct information on particle acceleration and coronal heating in the magnetically confined outer atmospheres. The structural relationship between the emission sources is mostly inferred from indirect arguments such as rotational modulation or gyroresonance emission. Direct VLBI provides evidence of resolved, extended coronae on solar-like stars.
\end{abstract}

\section{Introduction}

Coronal imaging in X-rays and microwaves has provided a tremendous amount of information on the structuring, the energy transport, and the stability of the corona of one star, our Sun. We may, however, expect a very different coronal structuring on a star that rotates ten times faster than the Sun, that has several percent of its surface covered with stellar spots, and that continually produces $100-1000$ times the X-rays and the microwaves the Sun radiates during strong flares.

I will discuss a few aspects of radio+X-ray studies of solar-like stars arguing that combined information from both regimes can provide some information on coronal structuring and energetics. I will confine this discussion to solar-type stars ( $\mathrm{F} / \mathrm{G} \mathrm{V}$ stars, in cases also $\mathrm{M}$ dwarfs). One of the obvious reasons for these stars to be important for us is the "solarstellar connection" that builds the direct bridge from solar knowledge to more exotic stellar systems. As for the less "solar-like" systems (e.g., RS CVn and Algol binaries, Ap stars) I refer to the review by Linsky (1994) 
and references therein, and to VLBI observations (e.g., Mutel et al. 1985).

Steady radio emissions from late-type active coronae can roughly be divided into i) gyroresonance emission from thermal, optically thick plasma in the lower, high- $B$ corona, and ii) gyrosynchrotron emission from accelerated, magnetically trapped electrons. Additionally, long-lasting coherent emissions are occasionally observed. Early observers (e.g., Linsky \& Gary 1983) showed that the quiescent radiation typically observed from Me dwarfs at frequencies of $1.5-5 \mathrm{GHz}$ is incompatible with gyroresonance radiation if $\mathrm{X}$-ray data are used to characterise the thermal plasma. Required source sizes are unreasonably large, or temperatures need to exceed considerably the electron temperatures determined from X-rays. Instead, $1-8 \mathrm{GHz}$ radio emission is typically nonthermal, with brightness temperatures exceeding $10^{8} \mathrm{~K}$ (White, Jackson, \& Kundu 1989). X-ray observations reveal very hot $(\geq 10 \mathrm{MK})$ quiescent plasma components in active stars, additionally to "cool" (1-3 MK) components otherwise dominating the coronae of inactive, old, and slowly rotating stars (such as the Sun).

\section{Gyrosynchrotron and X-ray emission: The role of flares?}

\subsection{OBSERVATIONS OF STARS}

The nonthermal quiescent radio and the thermal $\mathrm{X}$-ray luminosities of active main-sequence (MS) stars are linearly related, roughly $\log L_{\mathrm{X}} \approx \log L_{\mathrm{R}}+15.5$ over two orders of magnitude with a typical scatter by factors of 2-3 (Güdel et al. 1993; Fig. 1). This relation is the low-luminosity continuation of a similar relation for RS CVn binary systems (Drake, Simon, \& Linsky 1989; Fox et al. 1994) that also includes FK Com type stars, Algols, and some pre-main sequence stars (Güdel \& Benz 1993). The relation was successfully used to detect a series of new quiescent radio sources between spectral types F0V and late $\mathrm{K}$ based on the known $L_{\mathrm{X}}$, among them the first proxies of the Sun (Güdel et al. 1995abc).

\subsection{THE SOLAR ANALOGY}

The Sun supports considerable nonthermal gyrosynchrotron emission only during flaring episodes when electrons are accelerated up to $\mathrm{MeV}$ energies. At the same time, plasma is heated to several $10^{7} \mathrm{~K}$ for a short time. Remodeled solar flare observations reveal that the average flare luminosities $L_{\mathrm{X}}$ and $L_{\mathrm{R}}$ define the gross continuation of the quiescent stellar relation, although the overall trend differs from proportionality (slope $0.73 \pm 0.03$; see Fig. 1; Benz \& Güdel 1994). The trend for related coronal luminosities holds over 10 orders of magnitude, and suggests that i) particle acceleration arises along with the principal heating process in the active coronae considered 

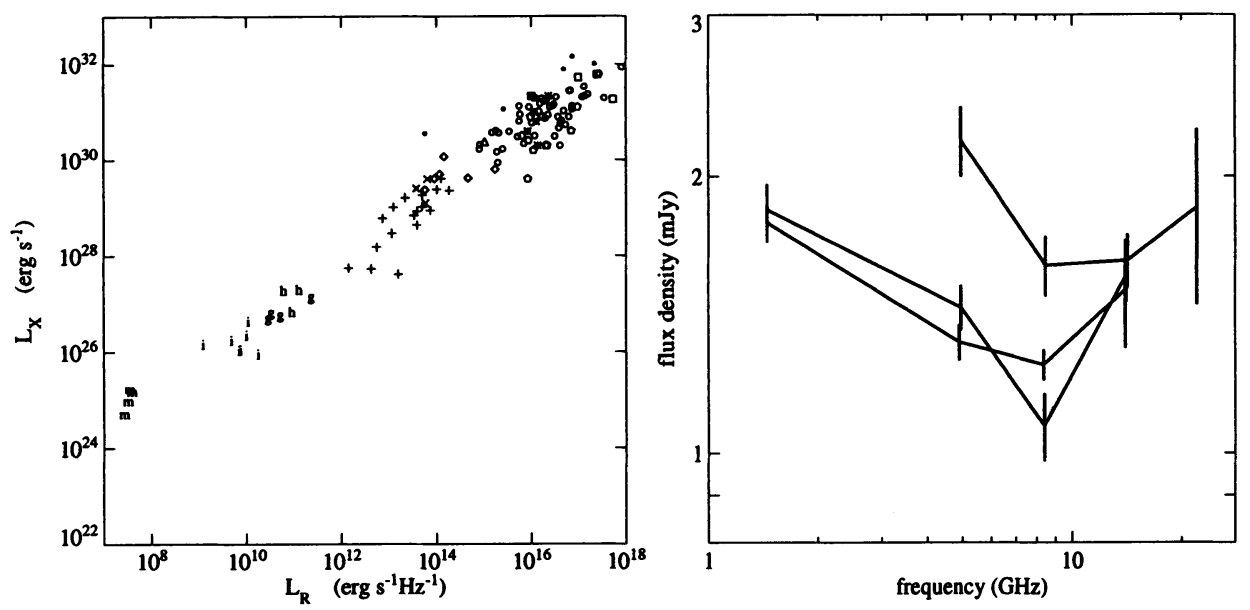

Figure 1. Two types of quiescent stellar radio emissions that are closely related with $\mathrm{X}$-ray emission. Left: Quiescent nonthermal 5-8 GHz radio luminosities of active stars plotted versus their X-ray luminosities. Symbols: + dMe stars; o RS CVn binaries; letters $\mathrm{g}, \mathrm{h}, \mathrm{i}, \mathrm{m}$ : solar flares (for other symbols, see Benz \& Güdel 1994). Right: Microwave spectra of UV Ceti, observed on three widely separated dates. The low-frequency portion is from gyrosynchrotron emission, while the high-frequency, increasing branch is compatible with gyroresonance emission from hot plasma. (From Güdel \& Benz 1995.)

here; ii) these processes are magnetic in origin, and iii) they are flare-like in character.

This suggests that at least parts of the "quiescent" coronae of active stars (the hotter plasma and the nonthermal electron population) share characteristics with solar flares. The cause for the relation between $L_{\mathrm{R}}$ and $L_{\mathrm{X}}$ (in solar flares and in quiescent stellar coronae) is unknown. In a solar flare model, accelerated electron populations heat the ambient plasma (by collisions) while the trapped high-pitch angle electrons emit gyrosynchrotron radiation. This model provides the correct time scales for slow variability in the quiescent emission (Güdel \& Benz 1993).

\section{Gyroresonance radio emission from the X-ray emitting corona}

In the quiet Sun, two thermal mechanisms emit observable radio emission: i) thermal free-free emission from chromospheric/transition region layers, and ii) gyroresonance emission from hot coronal plasma enclosed in strong magnetic fields above photospheric active regions. The second component is slowly variable depending on the distribution of sunspots. For active dwarfs, we expect significantly stronger gyroresonance radiation because, first, they have measured photospheric magnetic fields of several $\mathrm{kG}$ with filling factors often exceeding 50\% (Saar 1990), and second, magnetic field lines expand 
slowly (and thus magnetic flux densities decrease gently outwards) in an atmosphere that is nearly filled with radial field lines.

Under conditions in the low corona, gyroresonance emission remains optically thick beyond $10 \mathrm{GHz}$, and thus displays an increasing flux spectrum. In stars and in the flaring Sun, outer-corona nonthermal gyrosynchrotron emission typically becomes optically thin beyond $5-10 \mathrm{GHz}$ and from there decreases. The dM5.5e flare star UV Cet has repeatedly shown gyroresonance and gyrosynchrotron components in its radio spectra (Güdel \& Benz 1989, 1995; Fig. 1b). The brightness temperature,

$$
T_{\mathrm{B}}=\frac{S c^{2} d^{2}}{2 k \nu^{2} \pi R^{2}} \approx 2 \cdot 10^{7}\left(\frac{S}{1 \mathrm{mJy}}\right)\left(\frac{d}{1 \mathrm{pc}}\right)^{2}\left(\frac{1 \mathrm{GHz}}{\nu}\right)^{2}\left(\frac{R_{\odot}}{R}\right)^{2} \mathrm{~K}
$$

equals $T_{\text {eff }}$ for optically thick emission ( $S=$ measured radio flux density at Earth, $k=$ Boltzmann constant, $c=$ speed of light, $d=$ distance of the star, $R=$ source radius). For UV Cet with $T_{\text {eff }}=10^{7} \mathrm{~K}$ from X-ray observations, one finds $R \approx(1-2) R_{*}$ and hence, the low-corona filling factor $f_{\text {cor }} \approx 1$ for strong magnetic fields that confine hot plasma. We assume that the X-ray corona reaches out to a height $L$ with emission measure $\mathrm{EMX}_{\mathrm{X}}$. Then the radio emission at a given frequency $\nu$ will originate from a layer of radius $R \leq L+R_{*}$ and of thickness $l$ from within the $\mathrm{X}$-ray corona, comprising approximately the emission measure $\mathrm{EM}_{\mathrm{R}}=2 \pi R^{2} l n^{2}$. Hence

$$
\frac{l}{10 \Lambda_{\mathrm{B}}} \mathrm{EM}_{\mathrm{X}} \leq \mathrm{EM}_{\mathrm{R}} \approx \frac{l}{L} \mathrm{EM}_{\mathrm{X}} \leq \frac{l}{\Lambda_{\mathrm{B}}} \mathrm{EM}_{\mathrm{X}}
$$

where $\Lambda_{\mathrm{B}}$ is the magnetic scale height, and we conservatively assume $\Lambda_{\mathrm{B}} \leq$ $L \leq 10 \Lambda_{\mathrm{B}}$. Eq. (2) provides an upper and a lower limit for $n^{2} \Lambda_{\mathrm{B}}$. Further, $l$ is equal to the resonance length for gyromagnetic radiation, $l \approx \Lambda_{\mathrm{B}} \beta_{0}$, with $\beta_{0}=\left(2 k T /\left(m_{\mathrm{e}} c^{2}\right)\right)^{1 / 2}$. Since the bulk of the observed radiation corresponds to the highest harmonic $s$ of the gyrofrequency $\nu_{c}=e B /\left(2 \pi m_{\mathrm{e}} c\right)$ that is optically thick, the requirement for the optical depth

$$
\tau=l \kappa>2
$$

with the absorption coefficient $\kappa \sim \frac{n}{\nu} f(s, \theta, T)$ from Melrose (1985) provides a lower limit for $n \Lambda_{\mathrm{B}}$. For UV Cet, Eq. (2) and (3) confine the radiating harmonic to $s=4$ (marginally also 5), corresponding to "observable" magnetic field strengths of $1000-2000 \mathrm{G}\left(\nu_{\mathrm{obs}}=10-23 \mathrm{GHz}\right)$ with $f_{\text {cor }} \approx 1$; corresponding electron densities are $10^{9-11} \mathrm{~cm}^{-3}$ (Güdel \& Benz 1989).

Surprisingly, White, Lim, \& Kundu (1994) found that the detectability of the gyroresonance component is rather exceptional in a series of highfrequency observations of dMe stars. This is in particular true for the nearby 
analog of UV Cet, Proxima Centauri, for which Lim, White, \& Slee (1995) find $f_{\text {cor }}<0.10-0.15$ for high $-B$, hot $\left(10^{7} \mathrm{~K}\right)$ magnetic loops. It is likely that the hot material is either confined to small areas in the lower corona, or to larger heights where $B$ is much smaller (White et al. 1994).

As for earlier, more solar-like stars, a weak detection of the F5IV-V star Procyon could be due to thermal emission from the lower atmosphere, most probably free-free emission from the chromosphere/transition region (Drake, Simon, \& Brown 1993). The relative contribution of a possible gyroresonance component remains difficult to estimate, however.

\section{Thermal/nonthermal structure in solar-type stellar coronae}

\subsection{EVOLUTION OF THE HOT AND THE NONTHERMAL COMPONENTS}

In stars that are nearly identical to the Sun except for their rotation periods $P_{\text {rot }}$, the hotter coronal temperature $T_{\text {hot }}$ (from 2-T spectral fits) decays with increasing $P_{\text {rot }}$ (and thus age; "coronal cooling", Fig. 2), described by a power-law relation $L_{\mathrm{X}} \propto T_{\text {hot }}^{4}$ (Güdel \& Guinan 1995). The relative amount of hotter emission measure also decreases with age. Analogously, for radio emission in young MS stars, $L_{\mathrm{R}}$ decays from $\log L_{\mathrm{R}} \approx 14$ to $\log L_{\mathrm{R}}<13.4$ with $P_{\text {rot }}$ increasing by a factor of $\sim 2$ (Güdel et al. 1995a).

Since roughly $L_{\mathrm{R}} \propto L_{\mathrm{X}}$ for nonthermal radio emission from active stars, we find further evidence that steady nonthermal microwave emission predominantly occurs in those stars that support a very hot $X$-ray plasma component. Models for the X-ray temperature dependence on the rotation period have been given by Jordan \& Montesinos (1991).

\subsection{CORONAL STRUCTURING; A CASE STUDY}

2-D or 3-D coronal modeling has been performed for eclipsing binaries and rotationally modulated sources. For solar-type stars, the first method has been demostrated for $\alpha \mathrm{CrB}$ in which the eclipsing primary is an X-ray dark A star and the X-ray coronal source is an active G5V star (Schmitt \& Kürster 1994). Lightcurve modeling revealed a patchy distribution of active regions on the G star's surface and indicated that i) the X-ray coronal height must be very modest, i.e. $\sim 10^{10} \mathrm{~cm}$. ii) Derived electron densities are $n_{\mathrm{e}}>2.2 \cdot 10^{10} \mathrm{~cm}^{-3}$. Clearly, this structuring is reminiscent of solar active regions, but densities are significantly higher in this very active star.

The rotational modulation approach was applied to the proxy of the ZAMS Sun, EK Dra (Güdel et al. 1995b). Surprisingly, only the cooler plasma $(T \approx 2 \mathrm{MK})$ is rotationally modulated. A model-independent geometric estimate yields average cooler plasma densities $n_{\mathrm{e}} \geq 2.5 \cdot 10^{10} \mathrm{~cm}^{-3}$. For flares, densities about 10 times higher are derived. A height-dependent 

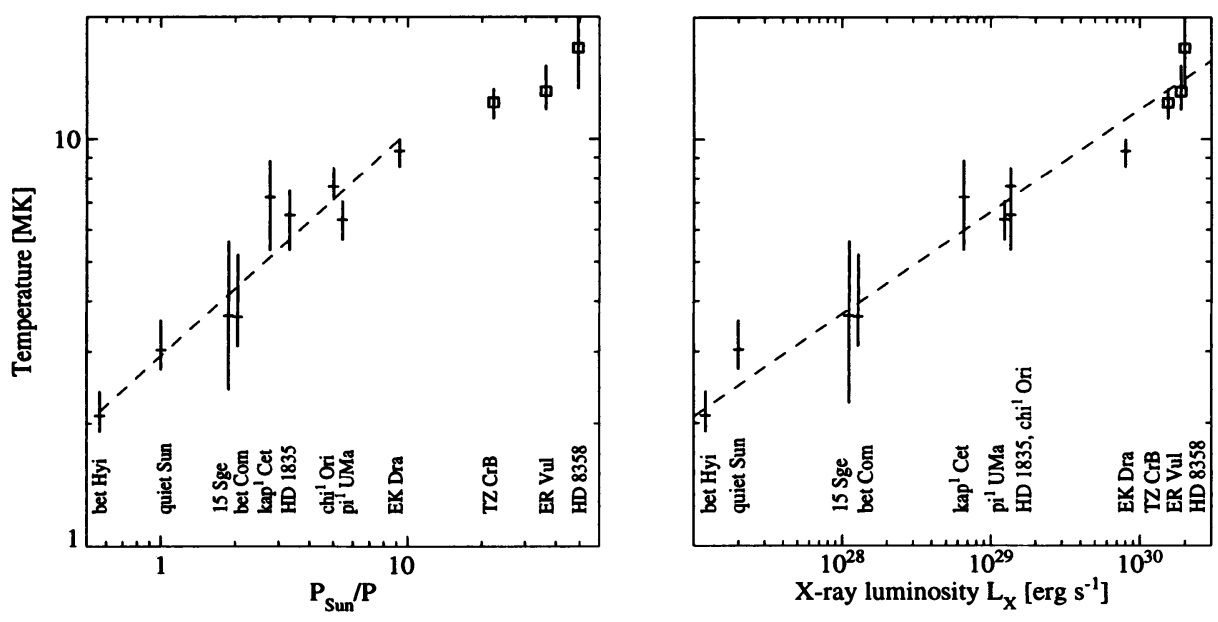

Figure 2. Left: Hotter ROSAT temperature $T_{\text {hot }}$ versus rotation rate for solar-like stars. Right: $T_{\text {hot }}$ versus total $L_{\mathrm{X}}$ for the same $\mathrm{G}$ star sample. - The rectangles represent tidally locked rapidly rotating binaries of two G stars. (After Güdel \& Guinan 1995.)

modeling of the "quiescent" X-ray sources shows that their height cannot exceed $0.2 R_{*}$, and the density reaches a minimum for $h \approx(0.10-0.15) R_{*}$, with $n_{\mathrm{e}} \approx 3.5 \cdot 10^{10} \mathrm{~cm}^{-3}$ (Fig. $3 \mathrm{~b}$ ). The maximum allowed extent of the cooler X-ray sources that contribute to the rotational modulation is shown in Fig. 3a for different model heights. In the framework of compact hydrostatic loops (Rosner, Tucker, \& Vaiana 1978) a consistent picture is obtained for a model with $n_{\mathrm{e}}=8 \cdot 10^{10} \mathrm{~cm}^{-3}$ and loop-top temperatures of $\sim 6 \cdot 10^{6} \mathrm{~K}$ with a height of $h=0.01 R_{*}$. The total heating rate, $E=9.8 \cdot 10^{4} p^{7 / 6}\left(\frac{\pi}{2} h\right)^{-5 / 6} \cdot V \approx 5 \cdot 10^{29} \mathrm{erg} \mathrm{s}^{-1}$ is then close to the observed radiative loss rate. A total of $10^{3-4}$ loops are required. Very similar results are obtained for the F0V star $47 \mathrm{Cas}$ (Güdel et al. 1995c). The compactness and the high densities imply that i) much of the increased coronal X-ray emission is due to high density rather than large volume; ii) the cooler $\mathrm{X}$-ray sources appear to be analogous to solar active regions, although at a more compact and denser level; iii) to confine the plasma, stronger average magnetic fields than in the solar case are required, since the thermal pressure must be balanced (a factor of $\sim 3$ for ten times higher density).

The absence of significant rotational modulation for the hotter component could imply that i) it is located at greater heights where stellar eclipses are less significant; ii) it is also located in low-lying areas in the corona, but distributed uniformly around the star; or iii) its location is at stellar latitudes that are not eclipsed by the star. The second model is problematical since, if the plasma resides in strong magnetic fields, gyroresonance radio emission would be detected at high frequencies. While this is poorly tested 

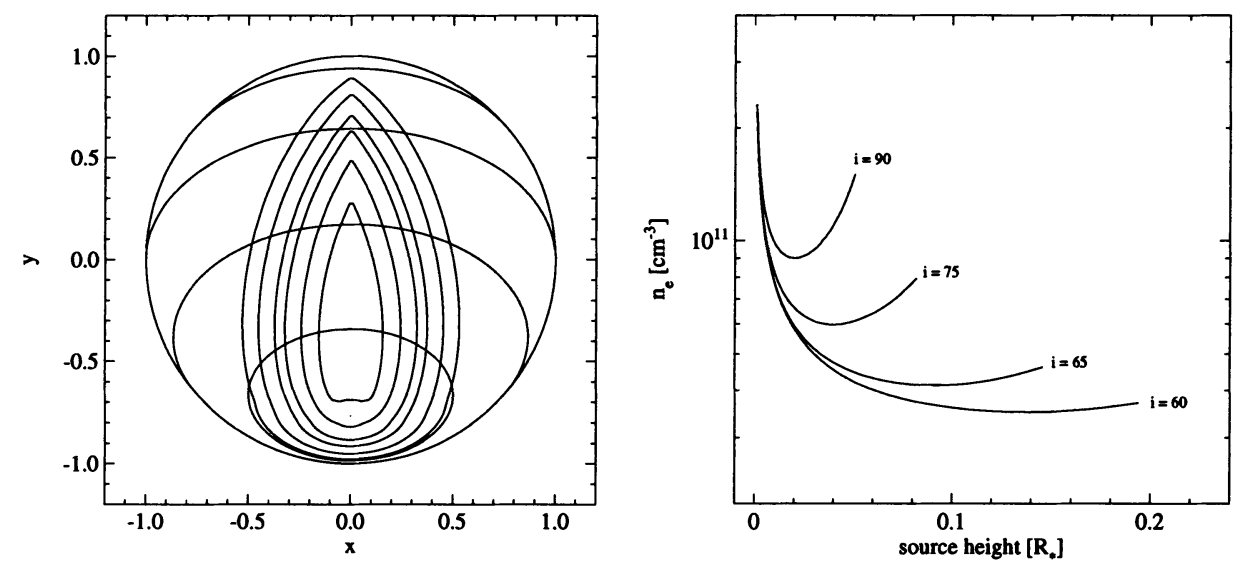

Figure 3. Left: Maximum source extent of an X-ray source (symmetric to a fixed meridian) compatible with the observed, modulated X-ray lightcurve of EK Dra. The distribution of individual sources within the outlined areas is left unspecified. Stellar inclination $i=60^{\circ}$. Latitude parallels are at $-60^{\circ},-30^{\circ}, 0^{\circ}$, and $+30^{\circ}$. Model source heights are, for decreasing source area, $h=0.001,0.01,0.03,0.05,0.1$, and $0.2 R_{*}$. Sources in the $\sim$ half-circles centered on the south pole remain invisible during the star's rotation. Right: Electron density $n_{\mathrm{e}}$ of the cooler X-ray plasma component in EK Dra as a function of the assumed geometric height $h$ of the emitting layer shown in the left figure, for different stellar inclinations $i$. (From Güdel et al. 1995b.)

for EK Dra, such a model is highly unlikely for the majority of cooler M dwarfs (Sect. 3).

How is the nonthermal component observed from EK Dra (Güdel et al. 1995b) related to the thermal plasma components? The lifetime of a $\mathrm{MeV}$ electron in a $n_{\mathrm{e}}=4 \cdot 10^{10} \mathrm{~cm}^{-3}$ plasma (the cooler component) is of the order of a minute, and much shorter for lower energies. To explain the steady radio emission, acceleration events must repeat on time scales much shorter than a minute. A limit is also set by the magnetic fields. If they reach $\mathrm{kG}$ strength, then the emission would be at too low harmonics to explain the observed brightness temperatures (see, e.g., White, Lim, \& Kundu 1994). Much more likely, the radio-emitting electrons reside at larger heights where densities are lower. This is one possibility also for the hotter thermal plasma component (see above), which appears to be an indicator for the presence of nonthermal particle populations (Sect. 4.1).

\section{Direct imaging by Very Long Baseline Interferometry (VLBI)}

For milli-arcsec resolution VLBI of active binary stars I refer to Mutel et al. (1985) as an example, and will again confine myself to main-sequence stars. Initial observations concentrated on $\mathrm{M}$ dwarfs for their relatively high 
fluxes and small distances (Benz \& Alef 1991; Benz, Alef, \& Güdel 1995). Brightness temperatures $T_{\mathrm{B}}>4 \cdot 10^{8} \mathrm{~K}$ were derived for $\mathrm{YZ} \mathrm{CMi,} \mathrm{a} \mathrm{direct}$ indication of the nonthermal character of this emission. EQ Peg B (dM6e) was observed during a giant radio flare. Its emission remained unresolved. The same is true for the dMe star AD Leo in quiescence. All observations show that the radio corona radius must be smaller than $1.8-1.9 R_{*}$, which corresponds to $\sim 2$ pressure scale heights of the hotter plasma component.

First significant resolution of an $M$ dwarf was obtained for the dM1e binary YY Gem (Alef, Benz, \& Güdel 1995), measured when the system was in conjunction, i.e. the optical stars eclipsed each other, while the radio coronae were superimposed. The source diameter is found to be 2.2 . $10^{11} \mathrm{~cm}$ or 2.4 stellar photospheric diameters, with $T_{\mathrm{B}} \approx 9 \cdot 10^{8} \mathrm{~K}$ supporting the nonthermal gyrosynchrotron model. This source size corresponds to a source stretching out to $\sim 2$ pressure scale heights of the hotter plasma above the photosphere.

\section{References}

Alef, W., Benz, A. O., \& Güdel, M. 1995, in preparation

Benz, A. O., \& Alef, W. 1991, A\&A, 252, L19

Benz, A. O., \& Güdel, M. 1994, A\&A, 285, 621

Benz, A. O., Alef, W., \& Güdel, M. 1995, A\&A, 298, 187

Drake, S. A., Simon, T., \& Linsky, J. L. 1989, ApJS, 71, 905

Drake, S. A., Simon, T., \& Brown, A. 1993, ApJ, 406, 247

Fox, D., et al. 1994, A\&A, 284, 91

Güdel, M., \& Benz, A. O. 1989, A\&A, 211, L5

Güdel, M., \& Benz, A. O. 1993, ApJ, 405, L63

Güdel, M., \& Benz, A. O. 1995, in Radio Emission from the Stars and the Sun. Eds. A. R. Taylor \& J. M. Paredes, in press

Güdel, M., \& Guinan, E. F. 1995, in Proceedings of IAU Coll. 152, ed. C. S. Bowyer, in press

Güdel, M., Schmitt, J. H. M. M., Bookbinder J. A., \& Fleming, T. S. 1993, ApJ, 405, 236

Güdel, M., Schmitt, J. H. M. M., \& Benz, A. O. 1995a, A\&A, in press

Güdel, M., Schmitt, J. H. M. M., Benz, A. O., \& Elias, N. M. II. 1995b, A\&A, 301, 201

Güdel, M., Schmitt, J. H. M. M., \& Benz, A. O. 1995c, A\&A, 293, L49

Jordan, C., \& Montesinos, B. 1991, MNRAS, 252, 21P

Lim, J., White, S. M., \& Slee, O. B. 1996, ApJ, in press

Linsky, J. L. 1994, in Solar Coronal Structures, IAU Coll. 144. Eds. V. Rusin, P. Heinzel, \& J.-C. Vial (Bratislava: Veda), 1

Linsky, J. L., \& Gary, D. E. 1983, ApJ, 274, 776

Melrose, D. B. 1985, in Solar Radiophysics. Eds. D. J. McLean \& N. R. Labrum (Cambridge: Cambridge University Press) 211

Mutel, R. L., Lestrade, J.-F., Preston, R. A., \& Phillips, R. B. 1985, ApJ, 289, 262

Rosner, R., Tucker, W. H., \& Vaiana, G. S. 1978, ApJ, 220, 643

Saar, S. H. 1990, in Solar. Photosphere: Structure, Convection, and Magnetic Fields. ed. J. O. Stenflo (Dordrecht: Reidel) 427

Schmitt, J. H. M. M., \& Kürster, M. 1994, Science, 262, 215

White, S. M., Jackson, P. D., \& Kundu, M. R. 1989, ApJS, 71, 895

White, S. M., Lim, J., \& Kundu, M. R. 1994, ApJ, 422, 293 